\title{
Sinus histiocytosis with massive
}

\section{Iymphadenopathy (Rosai-Dorfman disease) - imaging manifestations of renal} involvement

Sina Izadyar ${ }^{1}$, Farhad Samiei², Ali Gholamrezanezhad ${ }^{3,4}$

${ }^{1}$ Nuclear Medicine Department, Imam Khomeini Hospital Complex, Tehran University of Medical Sciences, Tehran, Iran ${ }^{2}$ Radiotherapy Department, Cancer Institute, Imam Khomeini Hospital Complex, Tehran University of Medical Sciences, Tehran, Iran ${ }^{3}$ Corresponding Author: Research Center for Nuclear Medicine, Shariati Hospital, Tehran University of Medical Sciences, Tehran, Iran

${ }^{4}$ University Hospitals of Case Western reserve University, Cleveland, $\mathrm{OH}, \mathrm{US}$

[Received 22 X 2013; Accepted 7 I 2014]

\begin{abstract}
A 23-year-old lady presented with abdominal fullness and distension as well as large abdominal masses in physical exam. Upon ultrasonographic evaluation, two large space occupying lesions anterior to kidneys, with no clear distinction from renal tissue, as well as bilateral hydronephrosis were found. The findings were confirmed by MRI. Histopathological analysis of renal masses and lymph nodes showed proliferation of histiocytes mixed with occasional multinucleated giant cells, immunostaining of which was positive for S-100 and CD68. We present renal scintigraphy features of this rare case of renal sinus histiocytosis with massive intraabdominal lymphadenopathy (Rosai-Dorfman disease).
\end{abstract}

KEY words: sinus histiocytosis, massive lymphadenopathy, Rosai-Dorfman disease, renal scintigraphy, ${ }^{99 m}$ Tc-DMSA, 99mTC-EC, magnetic resonance imaging

Nuclear Med Rev 2014; 17, 1: 44-46

\section{Case report}

A 23-year-old lady presented with abdominal fullness and distension as well as large abdominal masses in physical exam. Upon ultrasonographic evaluation, two large space occupying lesions anterior to kidneys, with no clear distinction from the renal tissue, as well as bilateral hydronephrosis were found. The findings were confirmed by MRI (Figure 1). ${ }^{99 m}$ Tc-DMSA renal scintigraphy (Figure 2) and ${ }^{99 m} \mathrm{Tc}-\mathrm{EC}$ renal scintigraphy (Figure 3) were also done to assess the renal function. Despite of significant scintigraphic abnormalities, both in static (DMSA) and dynamic (EC) renal scintigraphies, renal function in terms of blood urea nitrogen (BUN) $(34 \mathrm{mg} / \mathrm{dL})$ and serum creatinine $(0.5 \mathrm{mg} / \mathrm{dL})$ was normal.

Following percutaneous biopsy, histopathological analysis of renal masses and lymph nodes showed proliferation of histiocytes mixed with occasional multinucleated giant cells, im-

Correspondence to: Ali Gholamrezanezhad, MD, FEBNM

University Hospitals of Case Western reserve University, Cleveland

1034 Stonecutters Ln. South Euclid, OH, US 44121

Phone: 001 (443) 8397134

E-mail: a.gholamrezanezhad@yahoo.com

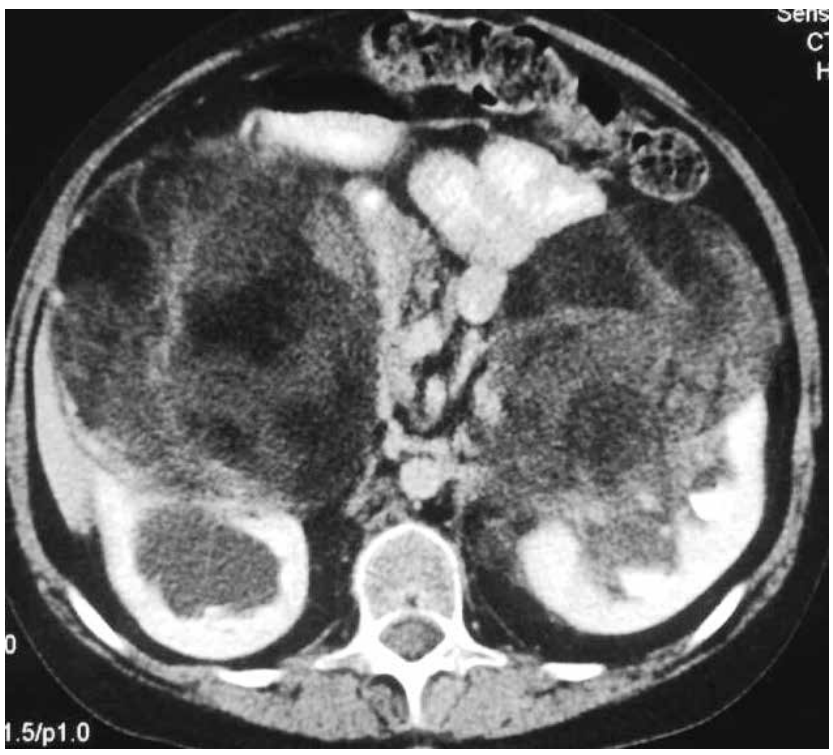

Figure 1. Magnetic Resonance Imaging (MRI) showed large space occupying lesions anterior to the kidneys. They showed compressive effect on renal pelvises and calyceal system, with secondary hydronephrosis 
A

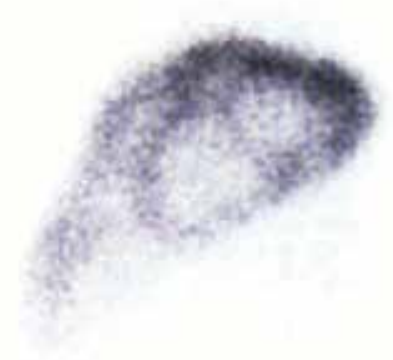

B

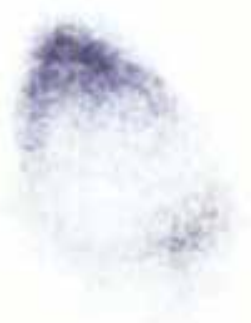

Figure 2. ${ }^{99 m}$ TC-DMSA renal scintigraphy in posterior (A) and anterior views (B) detected multiple abnormal photon-deficient areas in cortical regions of both kidneys, particularly in the mid-lower portions, indicating renal tissue involvement by histiocytosis. Anterior view shows significant attenuation of photons by the massive lymphadenopathies

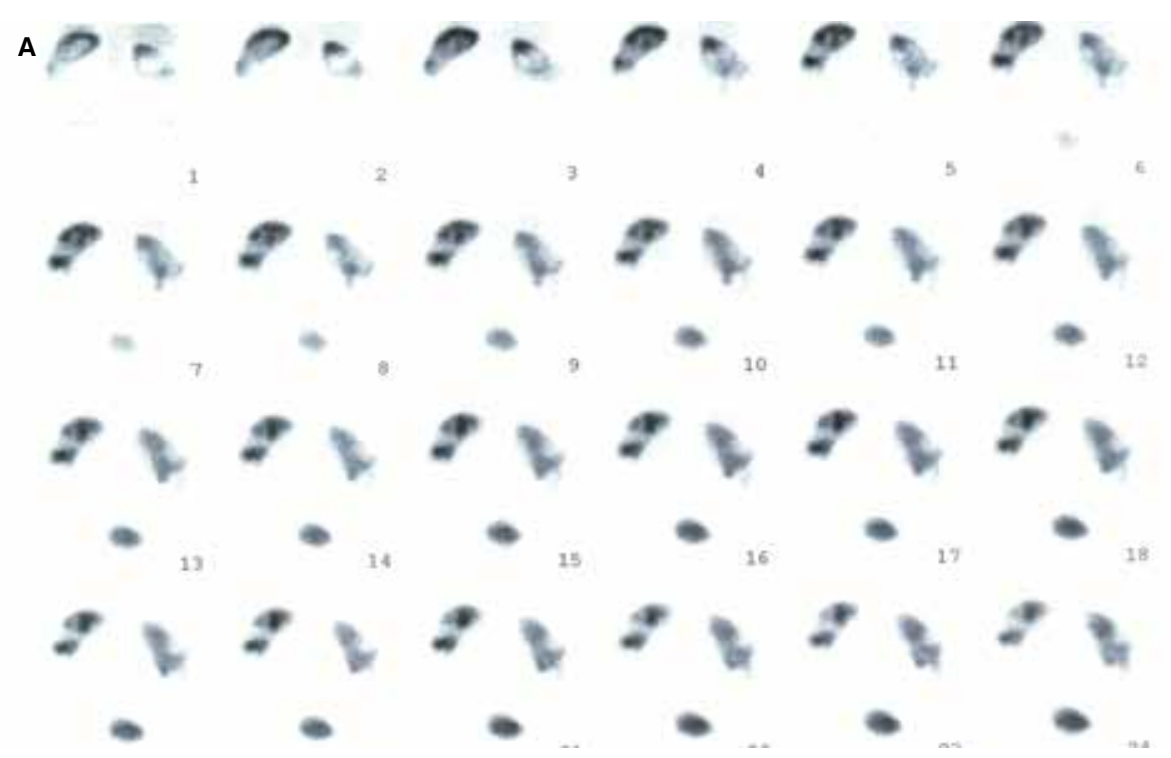

B
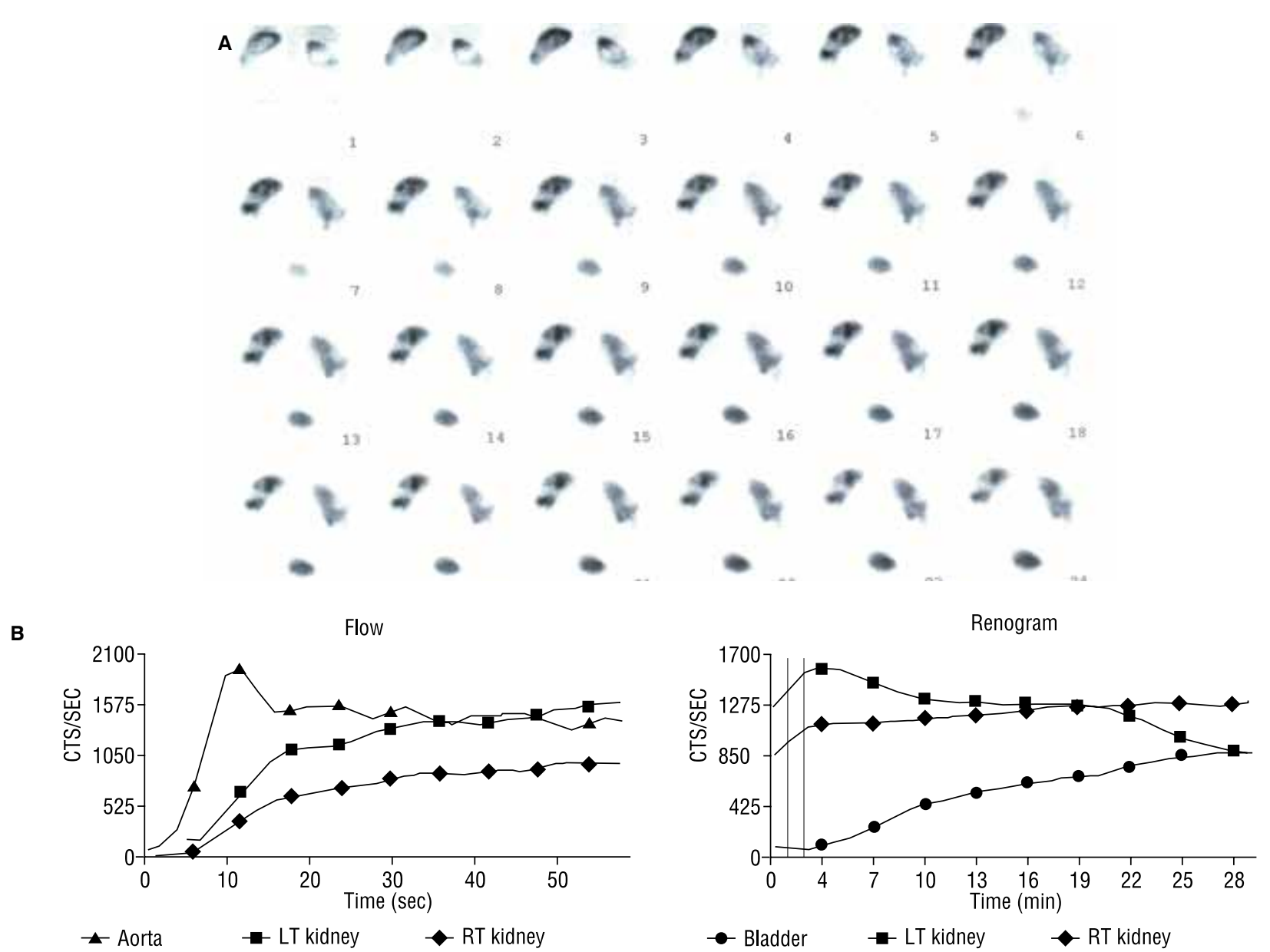

Figure 3. ${ }^{99 m}$ TC-EC renal scintigraphy (A) showed hypoperfused regions in both kidneys and cortical thinning involving the mid-and lower portions of both kidneys, as well as evidences of delayed radiotracer washout and partial retention, indicating hydronephrosis due to compressive effect by the extrarenal masses. Renogram (B) shows delayed washout of radiotracer from kidneys.

munostaining of which was positive for S-100 and CD68, so the diagnosis of renal sinus histiocytosis with massive intraabdominal lymphadenopathy (Rosai-Dorfman disease) was confirmed [1-3]. The patient was started on steroid treatment. After a year of steroid therapy, the patient was readmitted due to abdominal pain, found to have tumor growth.

Imaging features of Rosai-Dorfman disease, like the laboratory findings, are nonspecific. Ultrasound and MRI show soft-tissue 
space-occupying lesions, with no pathognomonic features. DMSA static renal scintigraphy shows renal cortical loss and dynamic renal scintigraphy shows reduced renal perfusion in the affected portions of kidneys. Imaging studies could potentially show significant parenchymal involvement, while laboratory studies are normal. So in patients with known Rosai-Dorfman disease, imaging studies are necessary, when there is a clinical suspicion for renal involvement, as laboratory studies seem to be not sensitive enough.

\section{References}

1. Rajasekharan C, Ratheesh NS, Nandinidevi R, Parvathy R. Rosai Dorfman disease: appearances can be deceptive. BMJ Case Rep 2012; 2012.

2. Romero Arenas MA, Singhi AD, Hruban RH, Cameron AM. Rosai-dorfman disease (sinus histiocytosis with massive lymphadenopathy) of the pancreas: third reported occurrence. J Gastrointest Cancer 2012; 43: 626-629.

3. Cooper SL, Jenrette JM. Rosai-Dorfman disease: management of CNS and systemic involvement. Clin Adv Hematol Oncol 2012; 10: 199-202. 